\title{
Theory and practice in visual interfaces for semi-structured document discovery and selection
}

\author{
Fernando Loizides ${ }^{\mathrm{a}, *}$, George Buchanan ${ }^{\mathrm{b}}$ and Keti Mavri ${ }^{\mathrm{c}}$ \\ ${ }^{\text {a }}$ University of Wolverhampton, Wolverhampton, UK \\ E-mail:fernando.loizides@wlv.ac.uk \\ ${ }^{\mathrm{b}}$ City University London, London, UK \\ E-mail: george.buchanan.1@city.ac.uk \\ ${ }^{\mathrm{c}}$ Cyprus University of Technology, Limassol, Cyprus \\ E-mail:aekaterini.mavri@cut.ac.cy
}

\begin{abstract}
With the increase in electronic publications, and indeed the availability of existing publications in digital form, as well as the encouragement of open access publication, comes a challenge. That challenge is to create assistive software to aid in the discovery and selection of relevant documents to one's information need. Visual interfaces have begun to address the need of information seekers in finding publications and wading through the large result sets that are returned from search engines. There is currently, little evidence to suggest that these interfaces are based on systematic research on requirements. In this article, we examine areas which contribute to the theory and practice of visual interfaces directly relating to the discovery and selection of publications. We bring together work from different fields in a targeted approach to assist the future creation of these interfaces.
\end{abstract}

Keywords: Electronic publishing, information seeking, interface interaction

\section{Introduction and motivation}

Publishing in academic journals and conferences has become faster, and easier with the ability to edit and submit documents electronically. With the increase of publications also come negative effects such as that of information overload and elevated discovery time of relevant resources. An information seeker often wades through several documents in order to find relevant publications utilizing generic search sources which network to several online repositories. Several tools have been created by researchers in order to assist the seekers in their visual academic document triage activities but very few have been successfully implemented in actual discovery of electronic publications. With electronic publishing increasing dramatically, we recognize the paramount importance for these tools to be improved and integrated within environments to assist the seekers. In this work, we present theoretical and practical work which encourages structured approaches to creating triage tools and improve the discovery process of electronic academic document publications.

\footnotetext{
*Corresponding author. E-mail: fernando.loizides@wlv.ac.uk.
} 
Document Selection is a process undergone by scholars, information professionals and information seekers daily to choose relevant documents on a topic. More recently, the term 'document triage' has been adopted to describe more accurately the document selection process. Document triage is the process by which an information seeker makes a relevance decision on a set of documents compared to his information need. An equivalent automated process is that of information retrieval. In turn, document triage and relevance decisions are part of the larger process of information seeking. Information seeking has been studied by many different disciplines.

Our main focus is the visual attention of information seekers during the document triage process. In particular, we are considering academic documents, which are semi-structured in nature. Knowledge that exists and contributes to the field of document triage is identified for a holistic picture of information seeking to be built. In order to familiarize ourselves with document triage and better understand the context within which our participants will be tested, we review research both in document triage, and within other directly related areas. We present the related work in a top down approach therefore also contributing an overview of information seeking while also covering a finer granularity of detail with relevant concepts such as satisficing. Specifically, the areas of relevance decisions, satisficing, document triage, eye-tracking, Cognitive psychology and human computer interaction principles are discussed.

\section{Relevance decisions}

Within information seeking, users need to identify documents ideally as being 'relevant' or 'not relevant'. The related work gives more detailed descriptions of the behaviors that our users are likely to perform and uncovers some likely reasons for this. The concept of relevance and relevance decisions is a difficult one to define $[55,68]$. Saracevic argues how the measure of relevance is 'of the effectiveness of a contact between a source and a destination in a communication process' [65]. Saracevic also states that 'relevance does not have to be explained; it is universally understood. It is an intuitive, primitive, yknow notion', in other words, 'people understand relevance intuitively' [66]. Saracevic's definitions, although not very technical, summarise the problem faced by systems in interpreting the documents retrieved as relevant or not for the information seeker. This is one of the major reasons why information retrieval systems, cannot presently substitute a human in making subjectively perfect relevance decisions. More definitions and explanations have been produced related to relevance decisions. One important distinction made, was that of separating a system's relevance decisions with that of a user [67]. Schamber et al. also give a concise discussion on the meaning of relevance [68].

There is also literature reporting on relevance from the system's perspective. As Park, building on Cooper's work on logical relevance [17] states, 'topical relevance is context-free and is based on fixed assumptions about the relationship between a topic of a document and a search question, ignoring an individual's particular context and state of needs' [43]. The focus can be set on the actual query terms which are used as a representation of the need and therefore, the interpretation of the query [34,77]. It was then that Froehlich suggested that there is no need to have one definition for relevance, but that information scientists 'can determine the diverse criteria that people bring to systems by which to judge its output' [25].

Relevance decisions (which can also called relevance ratings and relevance judgements) 'are based on information seekers' judgements about a document with respect to an actual problem' [51]. Most searches are mediated using electronic means. An information seeker will have in mind an information need, which he or she needs to satisfy (see section on Satisficing). In order to express this need to an 
electronic system, the information seeker must provide a representation of that need in a way that the system understands. Likely results are ranked and presented to the information seeker. In this way an information retrieval process is used to retrieve information which belongs to a fuzzy set [20], in that there are often not well defined boundaries regarding the relevance and information need. Information seekers may neglect relevant documents and conversely accept documents which upon further scrutiny provide very little information [10]. When performing document triage, relevance decisions are a direct result of the information seeker's visual search of a document. Cool et al. identified that seekers believe that 'Format Facets' affect their relevance decisions [16]. Furthermore, task complexity can also influence information seeking behavior and therefore the relevance decision process [13]. Bystrom and Jarvelin classified the types of information sources as (a) Fact-oriented: registers (manual and computerized catalogues and files) commercial databases; (b) Problem-oriented: the people concerned (for example, people proposing, or affected by, administrative actions) official documents (for example, agendas, meeting minutes, letters, applications, memoranda, maps, unpublished planning documents); and (c) Generalpurpose: experts (including knowledgeable colleagues), literature (for example, books, reports, journals, newspapers) personal collections (personal notes, calculations, etc.) They also classified the sources as being either internal or external to the organization in which the user works. The information seeking process however is not yet complete. The information seeker then has the final judgement on the perceived relevance of the documents to the information need.

\section{Satisficing}

Users, when making relevance decisions on documents, unlike automatic information retrieval systems, often do not attempt to make an optimal choice of documents. They do not read every word on every document and, after comparing all the documents, select the most mathematically probable relevant documents. This is evident in their behaviour, which is not always the same between users. This effect of making a decision between satisfying an information need in a way that success rather than attempting to find the optimal solution manually, has been dubbed 'satisficing' [74]. Information seekers will employ different searching strategies in order to obtain the required information. Satisficing has been well established within the information seeking field as representative behavior for users while searching for information, due to the time constraints and cognitive limitations [78]. 'It is logical that a user could not enter a library and carefully evaluate every book, periodical, and multimedia resource before selecting an item' [2]. We should note here that alternative approaches that support satisficing exist. For example, the model of a Generalized Information System [84] is also a theoretical construct which assumes that information seekers calculate the probability of the outcome of each decision to make an optimal decision. We do not follow these theoretical hypotheses and models since they do not accurately represent the process which we are studying. The GIS model, for example, would not be realistic in a situation which has thousands or perhaps millions of possible decision outcomes. For this reason, we follow the satisficing literature as a foundation framework of this research, which is also within the domain of information foraging and scent behavioral theories [60]. Foraging and information scent is a theory which states that humans will employ built-in searching strategies to seek out useful information just like searching for food for earlier humans. Reader and Payne [62] experiment with satisficing by giving a limited time to users in order to observe the effect of trying to obtain information that is 'good enough' rather than optimal. The authors hypothesize that as long as a document is 'good enough to meet the learning needs, it will be read'. They argue that satisficing would be more suitable when there 
are more than one document which is 'good enough' and if the differences among these texts are significant and perceptible. From these experiments they discovered that participants would use satisficing when the texts were not presented in an outline. It is interesting to also note that there was no evidence to show that the participants showed more efficient learning, in either of the searching strategies which may imply that satisficing is just as efficient as other methods such as sampling [75]. We note a possible gap in the research findings of Reader and Payne that could be expanded on. In their studies, they used texts between 1533 and 1672 words. This does not allow for reporting on behavior which is necessarily valid in longer (or shorter) text conditions. Duggan and Payne continue experiments on satisficing, reporting on skimming through texts with time constraints. They ask the question 'are readers able to allocate attention effectively when faced with a longer document than they can read in the time available?' [21]. The authors base their experimental motivation to the fact that 'there is little evidence that skim readers are able to devote their limited time to the most valuable parts of a single document'. They also present a hypothesis based on the Pirolli and Card's earlier work on foraging [60] which states that if 'readers are likely to begin to read linearly, then they may continue until the rate of information gain drops below the acceptable threshold. At this point they will leave the current patch of text and skip to the beginning of the next patch'. In [21], the authors focus their findings on understanding and memory rather than visual attention. Their data reported that memory for reading and efficiency, was not significantly worse in any of the two given conditions. Furthermore, their methodological approach allowed half of the users to read only half the paragraph contents rather than giving them the entire document. Behaviours however, such as the reader's 'tendency to glance across an entire page before settling to read' were reported using an eye-tracker. What is particularly interesting is that the experiment uncovered that skim readers where more likely to allocate their time to more important material within the text, even with time constraints were in place. Duggan and Payne presented further findings [22] from their eye-tracking study in a subsequent paper which highlighted some behavioural elements of the participants. They concluded that 'Locating information at the start of the paragraphs and pages increases the likelihood that it will be read'. In the same experiment they also report on how 'Information that is later in a text is still relevant to time-pressured readers'. In our research, we introduce further time constraint scenarios which add to the behavioral patterns reported here. We find that when skimming time is limited even further, visual and scrolling patterns vary even further.

\section{Document/information triage}

The word triage comes from the medical term meaning 'sorting and allocating aid on the basis of need for or likely benefit from medical treatment' (taken from Princeton on-line dictionary accessed 2015). This process can be applied to sorting information and specifically, wading through documents to assess their relevance to an information need. Research related to document triage as early as the 1960s has been reported on; previous to the terms' appearance. In order for us to have a consistent characterization of document triage in our research, we use the definition: 'Document Triage is the fast process by which information seekers, go through a set of potentially relevant documents to establish relevance to their information need'.

The document triage process has been described from related research in many ways. This literature either looks at the document triage process directly or provides indirect evidence through related studies. One example of this is Wang's cognitive model of document use [81]. Wang and Soergel describe document selection as 'he endpoint of a bibliographic search'. In this work the process takes a much wider approach than the one suggested in this paper, leaning towards the information seeking model 
as a whole. A narrower approach taken is that of Wacholder et al. where they describe the 'book selection process' as beginning 'when an individual has an information need that may be satisfied by a book (whether printed or electronic)' and ends with 'the user's decision as to whether the book contains enough information on the topic at hand that it merits more in-depth reading' [80]. This definition looks at the interaction of only one document (specifically a book type) from a within-document perspective.

The precise term 'document triage' is new to the digital library field and has been previously used in a limited number of work (NOTE: The term information document triage is NOT used in the way used by Lankes' model [61]. Lankes uses the term triage in the sense of directing users to expert sources which will later guide the information seeker and recommend likely useful material). Related terms however have been used to describe the process. We highlight some recurring phrases in the existing definitions of document triage which guide us in formulating our own definition. The term 'document triage' was first introduced in a broader scope as 'information triage' [52]. Here, Marshall et al. describe information triage as 'the process of sorting through relevant materials, and organizing them to meet the needs of the task at hand'. In later work Marshall et al. defined 'document triage' as 'the practice of quickly determining the merit and disposition of relevant documents' [5]. Another definition of document triage, defined the process as one 'determining important, time sensitive documents from unimportant ones' [44]. Marshall, in later work, talks about document triage as the process of 'sorting through large sets of documents to identify useful materials' [10]. Buchanan and Loizides define document triage as 'the critical point in the information seeking process when the user first decides the relevance of a document to their information need' [10]. As the process's workings became clearer, the term was refined to include the new findings. The definition for document triage was then defined as: 'the moment in the information seeking process when the user first decides the relevance of a document to their information need' [47]. The definitions above are analogous and complementary, since each is adapted to suit the individual research being performed.

A general model for document triage is presented in [48]. The model presents three stages at which document triage can occur. The first stage is that of the surrogate level (usually a results list). The second stage is that of within-document triage. A third stage, that is often neglected, is that of further reading triage. Spink et al., presents research into how information seekers triage results lists [76]. Their findings show how $70 \%$ of users will look at 2 pages of results lists or less before moving on to another query, thus dismissing the remaining document set. They also report on how 'users continue to have low tolerance for wading through large retrievals'. It is hypothesised that due to this low tolerance, users would find shortcuts to information seeking. Nicholas et al. reported that if an abstract webpage was accessed before the document could be downloaded, users would likely not download the remaining document [57]. They were not able to prove this theory however, do to subscription and access issues. Buchanan and Loizides however, were able to justify Nicholas et al. claims [10]. Beyond simply scanning through results, information seekers need appropriate interactions to facilitate their triage activities. Later research showed how categories (or piles) were used as a filing system for organizational document triage activities [10]. We realise how important the task of document triage support is since users 'are inundated with so much information that they spend the majority of their time sifting through documents rather than focusing on the task itself' [10]. From this evidence on organizing material during document triage, researchers have attempted to create predictive models on user interest. Badi et al., report on the organising and reading activities of information seekers during document triage, and how these activities can be used to detect user interest. The role organizational activities have on information seekers' triage process as well as relevance decisions is not a new concept. Furnas and Rauch developed 'design considerations for the construction of advanced information environments' [26]. 
Adler et al., piloted studies into the different reading styles of users by eliciting their reading behaviours using diary logs [1]. They found that there are there are different styles of reading, some of which are related directly to the document triage behaviour. Examples are: 'Reading in order to identify, Skimming, Reading to search/answer question, Reading to learn, Reading for cross-referencing'. We know from early work that users are more likely to consider reading on a screen as less affordable than reading on paper [10,39]. Interestingly, even earlier work contrasts the difference in affordance of reading on screens and paper. Some of the work shows results reporting on there being no difference between the two mediums $[3,68]$. Mills and Weldon present early work which reports on characteristics of the documents on electronic text and their effect on users' reading behavior [54]. It is reported that reading from electronic means has developed into a more 'shallower, fragmented, and less concentrated reading' [45]. This gives the hypothesis that when a user can spend less time and effort triaging through documents in an electronic environment he will do 'recent research, has suggested methods and design guidelines which can support the creation of effective electronic reading technologies' [58]. A similar study was conducted on graduate students, which investigated 'on how they extract and record information as they read' [59]. In their work, a model for document related activities is presented which identifies a read state and information review state. There are also other factors which look into the formulation of relevance decisions, both at a cognitive as well as a behavioural level. One such factor is topic familiarity. The level at which an information seeker is familiar with the subject area he or she is researching will affect the seeking and triage process [42]. Shipman et al. looks at annotations as criteria for deciding the relevance and usefulness of a passage within a document [72]. In this work, the focus is visual attention. The lessons learned from these studies are utilised when considering the methodology and data analysis, but are not further researched in depth.

\section{Visual attention and eye-tracking}

Document triage is a highly visual process. We are therefore required to look at work which identifies characteristics of documents, with regards to the relevance decision making process. Early work from Saracevic pioneered this research into the features which affect the decisions of users [64]. In this work, Saracevic investigates the effects of titles, abstracts and the full text has on participants' ratings of documents. Participants, after searching for documents, are returned first with the title, then the abstract and subsequently the full text of the document. They, at each point, provide a rating for the document based on the information they have. Interestingly, he reports how $78 \%$ of the evaluations had the same relevance rating. Marcus et al., presented a subsequent study in which participants were shown four document representations for each document, in a random order and asked to rate the document as to its perceived usefulness [7]. The participants were also asked to make the same judgement based on the full text of the document. In their findings Marcus et al. present the length hypothesis, in which the there is a positive correlation of a document representation with its length. 95\% of the participants indicated how titles and abstracts were useful to evaluating the documents. $74 \%$ predicted matching subject terms. Joseph Janes presented document representations to participants incrementally, using a different order each time to different groups, and asking them to assess the document on each representation [38]. The information presented was, the Main Title, Abstract, Bibliographic Citation and Indexing Terms. The abstract was reported as the most important feature, followed by the main title. The remaining two elements were reported as much less significant.

Cool et al. revisited factors on documents which have an effect on users' relevance decisions [16]. The main aim of the research was to see whether 'factors other than topical relevance are significant in such 
decisions'. One factor that they found was the format (or Formal characteristics of the document). Examples of these were: Lists, Diagrams, Statistics, Pictures; Class text; Book review; Title; Introduction; Division into topics. This information was extracted from the participants by use of questionnaires and suitable for the task. The visual attention by the participants here is subjective and can therefore neglect important elements of a document which are not exposed. Furthermore, the importance of each item is not specifically reported on. Carol L. Barry, in a later paper, investigates the reasons 'for pursuing or not pursuing documents based on information contained within representations of those documents (i.e., titles, abstracts, indexing terms, etc.)' [6]. Barry investigates three main document features; namely, document traits, source traits, and document representation. Document traits are defined as 'characteristics of the document that pertained to the physical format or actual publication of the document'. Source traits are defined as document characteristics that pertained to the intellectual source of the document. If a document trait or source trait was not explicitly mentioned, the response was simply coded for the document representation or part of the document representation. Barry found that only two characteristics, abstracts and titles, were marked by every respondent. Furthermore, only three document characteristics co-occurred with all three categories of responses: Abstracts, titles, and the full text of documents.

The vast majority of studies have focused on self-reported data. One of the main constraints in research thus far, has been extracting visual attention by observation rather than by self-reported evidence. Eyetracking is a suitable research methodology for this type of work. 'Eye-movement recordings can provide a dynamic trace of where ones attention is being directed in relation to a visual display' [41]. Using either a top-down or a bottom up approach, we can use eye-tracking to evidence 'how users make decisions and solve problems in search' and to identify which areas were 'read rather than scanned' [26]. We are then able to inform software creation by reporting on 'the sequence that users look at the visual elements in order to come to a decision' [19]. Eye-tracking records mainly, participants' fixation locations and timings. A 'higher fixation frequency on a particular area can be indicative of greater interest in the target, such as a photograph in a news report, or it can be a sign that the target is complex in some way and more difficult to encode' [35]. The duration of a fixation is also linked to the processingtime applied to the object being fixated [41]. Besides fixations, eye-tracking techniques record saccades and scan paths. Saccades "cannot tell us anything about the complexity or salience of an object in the interface' but regressive saccades (known also as backtracking eye-movements) can show us 'a measure of processing difficulty during encoding' [50]. Methodological development has been mostly tested on reading which takes place on a steady page (unlike our scrolling reader) and reports well on 'very small regressions, only skipping back two or three letters in reading tasks'. Equally, scan paths during a search can measure the time efficiency of a participant to reach a target [27]. In document triage, we are not aware of the user having a specific target and therefore cannot use this method to extract meaningful data at this point. Blink rates and pupil dilation 'can be used as an index of cognitive workload and effort' [8]. Surrounding elements which can affect a users' attention in a negative way, such as banners, have been reported on [11], but due to the nature of the documents we are reported in this work, these types of distractions should have minimal influential. We also recognise the capabilities and benefits of using eye-tracking from other studies which have been conducted successfully on similar documents, such as newspapers [56] and results lists [23].

\section{Cognitive psychology, perceptions and HCI principles}

The highly interactive process of document triage requires grounding on interaction principles as well as the cognitive understanding of information seekers. We need to be able to understand the cognitive 
limitations and natural interactions that users will have when presented with an information seeking task, in order to plan requirements and experiments strategically.

Document triage is a human-centered activity. Therefore, the internal workings of an individual's cognitive thinking needs to be acknowledged as a participatory factor in the decision making process. Marchionini addresses cognitive influence in the information seeking domain in that 'people construct and then draw on mental models to predict the effects of contemplated actions; that is, they make inferences based on running particular mental models' [3]. Here, we examine some basic cognitive psychology principles to help understand information seekers and users' behaviour in general. Users are also engaged with information and communication systems, while performing triage. The related field of human-computer interaction also provides a solid foundation by which to help identify problems and weaknesses in the systems being used. Cognitive psychology deals with the 'internal process involved in making sense of the environment' [24]. There are a series of categories which cognitive psychology studies. We are specifically interested in two of these: Experimental Cognitive Psychology and Computational Cognitive Science. The first category looks into experiments with healthy individuals within laboratory based scenarios. The second category, deals with the formulation of models to understand the human cognition. The main area in which this research reports on is users' behaviour in terms of visual attention. Although this is the main theme and focus of the thesis, it is important to remind the reader that there are also cognitive factors that can influence or constrain the document triage and decision making process. Human cognition is an important part of the triage process. Decisions are guided by principles of cognition which are influenced by a user's external world. One example of this is the user's working (short term) memory. An information seeker needs to devote attention, hold data in thought and also process visual data while performing triage. Baddeley introduces the components of memory which allow for these operations to take place [4]. Interestingly, when one of the components is required simultaneously for two tasks, the tasks will not be performed efficiently [63]. This principle is required for the effective design of software systems. A user should thus not be required to carry out tasks which require the same memory component simultaneously. Supporting systems can be designed to support the user in such activities. The ultimate purpose of the triage process is for the formulation of relevance decisions on documents. Decision making is a well-researched area in terms of the cognitive psychology of an individual. There are several theories into how decisions are formed and models which try to predict the decision outcomes of people; but as Hastie states: 'Most current decision theories are designed to account for the choice of one action at one point in time' [31]. It is difficult to accept the decision making principles found in these publications as the results comparing several studies have been controversial. The models that are therefore used to support the research into document triage are well established specialised models which have been tested and remain robust as to their conceptualisation of human behaviour. Human-computer interaction principles are guided heavily upon these cognitive guidelines. One example is the passing of information from sensory memory to short term memory (echoic memory). We can use these principles to ascertain that 'we are able to focus our attention selectively, choosing to attend to one thing rather than another' [79]. By using these principles, we can apply logical and semantic constraints [18] to better user interactions with prototype applications.

\section{Software tools}

Software tools can assist users in locating relevant information from a large data set. There is limited research in the tools which assist information seekers in their triage process. Wise et al., implement 
a visualization technique by employing spatial representations of large document sets [82]. Their aim was to create a visualization that may then be visually browsed and analyzed in ways that 'avoid language processing and that reduce the analysts' mental load'. In their research, they used Themescapes 'abstract, three dimensional landscapes of information that are constructed from document corpora' and Galaxies 'displaying cluster and document interrelatedness' to present the notion of document similarity. Although there was no formal user tests reported on the work provides insights of 'analysts' using the tool giving feedback of reduced time spent looking for relevant material. The users also report using the tool not just for document discovery but also for identifying document relationships (even if this is not a primary function of Themescapes). Another interpretation of a collected body of materials is presented by Marshall and Shipman [52]. In this research, a spatial hypertext tool is presented which allows information seekers to interpret results from documents and identify the structure of the document set. This is made feasible by the creation of objects, composites and collections, and allowing relationships to be defined. Building upon this early work, Shipman et al., created the Visual Knowledge Builder (VKB) $[70,71,73]$. VKB supports the 'incremental visual interpretation of information'. This tool was thoroughly utilized for collaborative efforts on shared information space. Similarly, a prototype tool called SketchTrieve, was also created to assist information and document triage [33]. SketchTrieve, was based on a conceptual model which followed the pattern: select the services you need, connect them, press Run, and results will be displayed. Another information visualization tool, created specifically for information triage, is TRIST (The Rapid Information Scanning Tool) [40]. TRIST is built on the analytical environment nSpace [83] and allows the 'rapid scanning over thousands of search results in one display, and includes multiple linked dimensions for result characterization and correlation'. TRIST allows for the information seeker to compare queries and find documents that are more tailored to their need. By doing this, document triage is informed by information that would have otherwise have taken multiple steps to achieve, all within one environment. Matching query terms to document content, like TRIST's attempt is important for information seekers. It helps them to relate their need to potentially relevant parts within a document. It is often hard however to locate the areas of the document which contain the query terms expressed by the user. A search engine will usually utilize the query terms in an information retrieval algorithm. Beyond that, there is usually no connection for the user, between the terms typed and the documents presented. Some users will use the Ctrl-F feature to find their terms within a document, but this is rarely the case [46]. It is evident that a more effective way to communicate the system's relevance decisions to the information seeker is needed. One way is to match up the query terms to areas within the documents. Currently, query terms are the established means by which an information seeker can make a request to a search engine. Directed browsing strategies can be assisted by several methods explained above using these terms, or variations of these, formulated by the user. Opportunistic search however, is also a big part of the information seeking process. It requires the triage of information in a less structured way. As it is becoming evident that 'keyword and hypertext cannot support all these new tasks well' more opportunistic and exploratory systems are being researched [9]. One such software tool uses Semantic fisheye views (SFEV's) to browser over collections with different metrics [36,37]. A similar approach was also implemented by Cockburn et al., this time, using space filling thumbnails with a zooming action to allow better space real estate [15]. Screen real estate is one of the limitations that challenge the above prototypes. The question asked by Bae et al. was whether different display types, would have an effect on the way users perform document triage [5]. In their findings they report how there are more transitions using multiple displays rather than a single display 'Additionally, users evaluated documents more by reading their contents and less often relied solely on metadata. Users spent more time reading and interacting with documents that they valued'. This corresponds with the 
finding that reading time correlates with assessing document value in the triage field [14]. A common approach to supporting users' triage activities is by enriched visual interfaces using scroll bars (or any bars representing the document length). Two software tools, FindSkim and ProfileSkim, created visualization in the task bars, to indicate the location of query terms in a document [28-30]. ProfileSkim, also added bar charts to allow the user to find heavily populated areas, query terms wise, within the document. A similar basis was used by Donald Byrd, who used color and term highlighting scrollbars [12] and Schwartz et al. who used term distribution visualizations [69]. The argument for making use of text structure when retrieving from full text documents has also been investigated by Marti A. Hearst, and a prototype 'a visualization paradigm, called TileBars' is presented to aid the information seeker [32]. The same information and principle as Hendry and Harper [33] was implemented with some additions, such as snippets for reading the results before navigating towards the related area. This method was favorable with participants. Query term matching has also been used in SmartFind, another hybrid Ctrl/Cmd-F type tool which uses Term Frequency $\times$ Inverse Document Frequency (TFIDF) algorithms within a document to provide potentially significant document areas to the information seeker [46]. TriDoc is a bespoke document triage tool which combines the high level results list view of document results with within-document scanning and information searching [49]. Currently, there are two interfaces supported by TriDoc. Both prototypes are hosted in a single-screen interface that integrates surrogate as well as within-document views; as well as snippets of individual sections of the document, combined with a full-text reading pane. This approach follows the research not on visualization presentation but on the visual attention of users; a bottom approach unlike the other presented tools. The interface allows for a 'natural' linear type scanning of the document contents to happen in a non-linear fashion and minimizes scrolling. TriDoc accommodated faster triage from the users test and received positive feedback from the information seekers (note: one interfaces received a higher rating than the other). TriDoc is an ongoing project which is currently under development, unlike many of the reported tools in this article [53].

\section{Summary}

This article was written in order to bring together work from various areas in an attempt to equip interface designers with the required theory and practice to create grounded decisions on visual requirements for the discovery and selection process of electronic publications. Although not exhaustive, the work covers the foundations required for the specialized field and includes work from the domains of relevance decisions, satisficing, document triage, eye-tracking, Cognitive psychology and human-computer interaction.

\section{References}

[1] A. Adler, A. Gujar, B.L. Harrison, K. O'Hara and A. Sellen, A diary study of work-related reading: Design implications for digital reading devices, in: Proceedings of the SIGCHI Conference on Human Factors in Computing Systems, 1998, pp. 241-248.

[2] D.E. Agosto, Bounded rationality and satisficing in young people's web-based decision making, Journal of the American Society for Information Science and Technology 53 (2002), 16-27.

[3] S. Askwall, Computer supported reading vs reading text on paper: A comparison of two reading situations, International Journal of Man-Machine Studies 22(4) (1985), 425-439.

[4] A.D. Baddeley, Is working memory still working?, American Psychologist 56 (2001), 851-864. 
[5] S. Bae, R. Badi, K.A. Meintanis, J.M. Moore, A. Zacchi, H. Hsieh, C.C. Marshall and F.M. Shipman III, Effects of display configurations on document triage, in: INTERACT, 2005, pp. 130-143.

[6] C.L. Barry, Document representations and clues to document relevance, Journal of the American Society for Information Science 49(14) (1998), 1293-1303.

[7] K.P. Benenfeld, A.R. Marcus and S. Richard, Catalog information and text as indicators of relevance, Journal of the American Society for Information Science 29(1) (1978), 15-30.

[8] D. Bruneau, M.A. Sasse and J.D. McCarthy, The eyes never lie: The use of eye tracking data in HCI research, in: Proceedings of the CHIO2 Workshop on Physiological Computing, 2002, pp. 441-480.

[9] D. Bryan and A. Gershman, Opportunistic exploration of large consumer product spaces, in: Proceedings of the 1st ACM Conference on Electronic Commerce, 1999, pp. 41-47.

[10] G. Buchanan and F. Loizides, Investigating document triage on paper and electronic media, in: European Conf. on Research and Advanced Technology for Digital Libraries, 2007, pp. 416-427.

[11] M. Burke, A. Hornof, E. Nilsen and N. Gorman, High-cost banner blindness: Ads increase perceived workload, hinder visual search, and are forgotten, ACM Trans. Computer-Human Interaction 12(4) (2005), 423-445.

[12] D. Byrd, A scrollbar-based visualization for document navigation, in: Proceedings of the Fourth ACM Conference on Digital Libraries, 1999, pp. 122-129.

[13] K. Bystrom and K. Jarvelin, Task complexity affects information seeking and use, Information Processing and Management 31(2) (1995), 191-213.

[14] P.K. Chan, A non-invasive learning approach to building web user profiles, 1999.

[15] A. Cockburn, C. Gutwin and J. Alexander, Faster document navigation with space-filling thumbnails, in: Proceedings of the SIGCHI Conference on Human Factors in Computing Systems, 2006, pp. 1-10.

[16] C. Cool, N.J. Belkin, O. Frieder and P. Kantor, Characteristics of texts affecting relevance judgments, in: Proceedings of the 14th National Online Meeting, 1993, pp. 77-84.

[17] W.S. Cooper, A definition of relevance for information retrieval, Information storage and retrieval 7(1) (1971), $19-37$.

[18] N. Donald, The Psychology of Everyday Things, Basic Books, 1988.

[19] D. Drieghe, K. Rayner and A. Pollatsek, The mind's eye: Cognitive and applied aspects of eye movement research, Journal of Experimental Psychology. Human Perception and Performance 5(1) (2003), 954-959.

[20] D. Dubois, H. Prade and R.R. Yager, Readings in Fuzzy Sets for Intelligent Systems, The Morgan Kaufmann Series in Representation and Reasoning, Morgan Kaufmann Publishers, 1993.

[21] G.B. Duggan and S.J. Payne, Skim reading by satisficing: Evidence from eye tracking, in: SIGCHI Conference on Human Factors, 2009, pp. 228-242.

[22] G.B. Duggan and S.J. Payne, Text skimming: The process and effectiveness of foraging through text under time pressure, in: CHI Conference on Human Factors in Computing Systems, 2011, pp. 1141-1150.

[23] S.T. Dumais, G. Buscher and E. Cutrell, Individual differences in gaze patterns for web search, in: Proceeding of the Third Symposium on Information Interaction in Context, 2010, pp. 185-194.

[24] M.W. Eysenck and M.T. Keane, Cognitive Psychology: A Student's Handbook, 2000.

[25] T.J. Froehlich, Relevance reconsidered towards an agenda for the 21st century: Introduction to special topic issue on relevance research, Journal of American Society for Information Science 45(3) (1994), 124-134.

[26] G.W. Furnas and S.J. Rauch, Considerations for information environments and the navique workspace, in: Proceedings of the Third ACM Conference on Digital Libraries, 1998, pp. 79-88.

[27] H.J. Goldberg and X.P. Kotval, Computer interface evaluation using eye movements: Methods and constructs, International Journal of Industrial Ergonomics 24 (1999), 631-645.

[28] D.J. Harper, S. Coulthard and S. Yixing, A language modelling approach to relevance profiling for document browsing, in: Proceedings of the 2nd ACM/IEEE-CS Joint Conference on Digital Libraries, JCDL'02, 2002, pp. 76-83.

[29] D.J. Harper, I. Koychev and Y. Sun, Query-based document skimming: A user-centred evaluation of relevance profiling, in: Proceedings of the 25th European Conference on IR Research, ECIR'03, 2003, pp. 377-392.

[30] D.J. Harper, I. Koychev, Y. Sun and I. Pirie, Within-document retrieval: A user-centred evaluation of relevance profiling, Information Retrieval 7(3) (2004), 265-290.

[31] R. Hastie, Problems for judgment and decision making, Annual Review of Psychology 52 (2001), 653-683.

[32] M.A. Hearst, Tilebars: Visualization of term distribution information in full text information access, in: Proceedings of the SIGCHI Conference on Human Factors in Computing Systems, 1995, pp. 59-66.

[33] D.G. Hendry and D.J. Harper, An informal information-seeking environment, Journal of American Society for Information Science 48(11) (1997), 1036-1048.

[34] D.L. Howard, Pertinence as reflected in personal constructs, Journal of American Society for Information Science 45(3) (1994), 172-185.

[35] R.J.K. Jacob and K.S. Karn, Eye tracking in human-computer interaction and usability research: Ready to deliver the promise, Cognitive Psychology 8 (1976), 441-480. 
[36] P. Janecek and P. Pu, Opportunistic search with semantic fisheye views, in: Web Information Systems WISE, 2004, pp. 668680.

[37] P. Janecek and P. Pu, An evaluation of semantic fisheye views for opportunistic search in an annotated image collection, International Journal on Digital Libraries 5(1) (2005), 42-56.

[38] J.W. Janes, Relevance judgments and the incremental presentation of document representations, Information Processing and Management 27(6) (1991), 629-646.

[39] M. Jones, G. Buchanan and N. Mohd, An evaluation of WebTwig - A site outliner for handheld web access, in: Proceedings of the 1st International Symposium on Handheld and Ubiquitous Computing, 1999, pp. 343-345.

[40] D. Jonker, D. Schroh, B. Wright, P. Proulx and B. Cort, Information triage with trist, in: Conference on Intelligence Analysis, 2005, pp. 2-4.

[41] M.A. Just and P.A. Carpenter, Eye fixations and cognitive processes, Cognitive Psychology 8 (1976), 441-480.

[42] D. Kelly and C. Cool, The effects of topic familiarity on information search behavior, in: Proceedings of the 2nd ACM/IEEE-CS Joint Conference on Digital Libraries, 2002, pp. 74-75.

[43] T. Kim Park, Toward a theory of user-based relevance: A call for a new paradigm of inquiry, Journal of American Society for Information Science 45(3) (1994), 135-141.

[44] K.L. Kwok, P. Deng, N. Dinstl, H.L. Sun, W. Xu, P. Peng and J. Doyon, CHINET: A Chinese name finder system for document triage, in: Poster in Conference on Intelligence Analysis, 2005.

[45] D.M. Levy, I read the news today, oh boy: Reading and attention in digital libraries, in: Proceedings of the Second ACM International Conference on Digital Libraries, DL'97, 1997, pp. 202-211.

[46] F. Loizides and G. Buchanan, The myth of find: User behavior and attitudes towards the basic search feature, in: Joint Conference on Digital Libraries, 2008, pp. 48-51.

[47] F. Loizides and G. Buchanan, An empirical study of user navigation during document triage, in: Proceedings of the 13th European Conference on Research and Advanced Technology for Digital Libraries, 2009, pp. 138-149.

[48] F. Loizides and G.R. Buchanan, Towards a framework for human (manual) information retrieval, in: Multidisciplinary Information Retrieval, 2013, pp. 87-98.

[49] F. Loizides, T. Photiades, A. Mavri and P. Zaphiris, On interactive interfaces for semi-structured academic document seeking and relevance decision making, New Rev. Inf. Networking 2(19) (2014), 67-95.

[50] M. Macleod, Usability Evaluation in Industry: Performance Measurement and Ecological Validity, Taylor and Francis, London, 1995.

[51] G. Marchionini, Information Seeking in Electronic Environments, Cambridge Univ. Press, 1995.

[52] C.C. Marshall and F.M. Shipman III, Spatial hypertext and the practice of information triage, in: Proceedings of the Eighth ACM Conference on Hypertext, 1997, pp. 124-133.

[53] A. Mavri, F. Loizides, T. Photiadis and P. Zaphiris, We have the content. .. now what?, Information Design Journal 20(3) (2013), 247-265.

[54] C.B. Mills and L.J. Weldon, Reading text from computer screens, ACM Computing Surveys (CSUR) 19(4) (1987), 329357.

[55] S. Mizzaro, Relevance: The whole history, Journal of the American Society for Information Science 48(9) (1997), 810832 .

[56] M. Mosconi, M. Porta and A. Ravarelli, On-line newspapers and multimedia content: An eye tracking study, in: Proceedings of the 26th Annual ACM International Conference on Design of Communication, 2008, pp. 55-64.

[57] D. Nicholas, P. Huntington, H.R. Jamali and A. Watkinson, The information seeking behavior of the users of digital scholarly journals, Information Processing and Management 42(5) (2006), 1345-1365.

[58] K. O'Hara and A. Sellen, A comparison of reading paper and on-line documents, in: Proceedings of the SIGCHI Conference on Human Factors in Computing Systems, 1997, pp. 335-342.

[59] K. O'Hara, F. Smith, W. Newman and A. Sellen, Student readers' use of library documents: Implications for library technologies, in: Proceedings of the SIGCHI Conference on Human Factors in Computing Systems, 1998, pp. $233-240$.

[60] P. Pirolli and S. Card, Information foraging, Psychological Review 106(4) (1999), 643-675.

[61] J. Pomerantz, S. Nicholson and R.D. Lankes, Digital reference triage: An investigation using the Delphi method into the factors influencing question routing and assignment, The Library Quarterly 73(2) (2006), 103-120.

[62] W.R. Reader and S.J. Payne, Allocating time across multiple texts: Sampling and satisficing, Human-Computer Interaction Journal 22(3) (2007), 263-298.

[63] T.W. Robbins, E.J. Anderson, D.R. Bradley, A.C. Barker, C. Fearnyhough, R. Henson, S.R. Hudson and A. Baddeley, Working memory in chess, Memory and Cognition 24(1) (1996), 83-89.

[64] T. Saracevic, Comparative effects of titles, abstracts and full text on relevance judgments, Journal of the American Society for Inf. Science 6(1) (1969), 126-139.

[65] T. Saracevic, Relevance: A review of and a framework for the thinking on the notion in information science, Journal of the American Society for Information Science 26(6) (1975), 321-343. 
[66] T. Saracevic, Relevance reconsidered 96. Information science: Integration in perspective, in: Proceedings of Second International Conference on Conceptions of Library and Information Science, 1996, pp. 201-218.

[67] L. Schamber, Relevance and information behavior, Annual Review of Information Science and Technology (ARIST) 29 (1994), 3-48.

[68] L. Schamber, M. Eisenberg and M.S. Nilan, A re-examination of relevance: Toward a dynamic, situational definition, Information Processing and Management 26(6) (1990), 755-776.

[69] M. Schwartz, C. Hash and L.M. Liebrock, Term distribution visualizations with focus+context, in: Proceedings of the 2009 ACM Symposium on Applied Computing, 2009, pp. 1792-1799.

[70] F. Shipman, R. Airhart, H. Hsieh, P. Maloor, J.M. Moore and D. Shah, Visual and spatial communication and task organization using the visual knowledge builder, in: Proceedings of the 2001 International ACM SIGGROUP Conference on Supporting Group Work, 2001, pp. 260-269.

[71] F. Shipman, J.M. Moore, P. Maloor, H. Hsieh and R. Akkapeddi, Semantics happen: knowledge building in spatial hypertext, in: Proceedings of the Thirteenth ACM Conference on Hypertext and Hypermedia, 2002, pp. 25-34.

[72] F. Shipman, M. Price, C.C. Marshall and G. Golovchinsky, Identifying useful passages in documents based on annotation patterns, in: Proceedings of the European Conference on Digital Libraries, 2003, pp. 101-112.

[73] F.M. Shipman III, H. Hsieh, P. Maloor and J.M. Moore, The visual knowledge builder: A second generation spatial hypertext, in: Proceedings of the 12th ACM Conference on Hypertext and Hypermedia, 2001, pp. 113-122.

[74] H.A. Simon, Rational choice and the structure of the environment, Psychological Review 63 (1956), 129-138.

[75] L.K. Son and J. Metcalfe, Metacognitive and control strategies in study-time allocation, Journal of Experimental Psychology: Learning, Memory, and Cognition 26(1) (2000), 204-221.

[76] A. Spink, B.J. Jansen, D. Wolfram and T. Saracevic, From e-sex to e-commerce: Web search changes, COMPUTER 35(3) (2002), 107-109.

[77] D.R. Swanson, Subjective versus objective relevance in bibliographic retrieval systems, Library Quarterly 56(4) (1986), 389-398.

[78] T. Tyszka, Information and evaluation processes in decision making, in: Social and Moral Values: Individual and Societal Perspectives, 1989, pp. 175-193.

[79] J. von Neumann and O. Morgenstern, Theory of Games and Economic Behavior, Princeton Univ. Press, 1944.

[80] N. Wacholder, L. Liu and Y. Liu, Selecting books: A performance-based study, in: Proceedings of the 6th ACM/IEEE-CS Joint Conference on Digital Libraries, 2006, p. 337.

[81] P. Wang and D. Soergel, A cognitive model of document use during a research project: Study in document selection, Journal of the American Society for Information Science 49(2) (1998), 115-133.

[82] J.A. Wise, J.J. Thomas, K. Pennock, D. Lantrip, M. Pottier, A. Schur and V. Crow, Visualizing the non-visual: Spatial analysis and interaction with information from text documents, in: Proceedings of the 1995 IEEE Symposium on Information Visualization, 1995, pp. 51-58.

[83] W. Wright, D. Schroh, P. Proulx, A. Skaburskis and B. Cort, Advances in nSpace - The sandbox for analysis, in: Poster at Conference on Intelligence Analysis, 2005.

[84] M.C. Yovits and C.R. Foulk, Experiments and analysis of information use and value in a decision-making, Journal of the American Society for Information Science 36(2) (1985), 63-81. 